\title{
THE VECTORCARDIOGRAM OF MITRAL STENOSIS
}

\author{
BY \\ JOHN SHILLINGFORD * AND WALLACE BRIGDEN
}

From the Cardiac Department of the London Hospital

Received September 2, 1953

Right ventricular preponderance and rotation of the heart specially influence the electrocardiogram of mitral stenosis, but it is often difficult to apportion their separate influence. This paper describes some of the abnormalities of the vectorcardiogram in mitral stenosis and relates them to the clinical and electrocardiographic findings.

Methods. Twenty patients with mitral stenosis were investigated. None showed clinical evidence of other valve lesions. A detailed clinical assessment of all cases was made and in some the levels of pulmonary artery and right ventricular pressures were determined by cardiac catheterization. Electrocardiography included standard limb leads, unipolar limb leads, chest leads V1 to $\mathrm{V} 7$, and in some patients V4R and V6R. The three plane projections of the spatial vectorcardiogram were obtained by the method previously described (Brigden and Shillingford, 1952). Fourteen of these patients were being considered for mitral valvotomy; hence most had severe symptoms, small hearts, and evidence of pulmonary hypertension.

\section{RESULTS}

The vectorcardiogram showed a considerable range of abnormality which could be broadly related to the clinical state of the patients. No significant abnormality was present in the vectorcardiograms obtained from three patients who had no symptoms, no general cardiac enlargement (left auricles were slightly large), nor evidence of pulmonary hypertension at rest (Fig. 1A). The vectorcardiograms from three patients with severe cardiac failure and great enlargement of the heart. also showed relatively slight abnormalities (Fig. 1B); the horizontal projection showed a recession of the first part so that the whole loop was narrowed in this plane. Right ventricular preponderance. and rotation were not seen.

The other 14 patients had evidence of moderate or severe pulmonary hypertension at rest with slight to moderate enlargement of the heart without congestive cardiac failure. All of these showed abnormalities of the vectorcardiogram and further observations relate to these patients unless. otherwise stated. Two examples are shown in Fig. 1, C and D.

The frontal loops showed varying degrees of rotation and deviation to the right; in each a line was. drawn through zero potential to the most distant point of the loop to represent the greatest vector in the frontal plane. Fig. 3 shows these axes for the whole series and it is apparent that those from patients in this group show the greatest deviation to the right and the more vertical position. Many of the frontal loops in this group show a further abnormality in the deviation of the later part of the loop into the right, lower and upper quadrants (Fig. 2F).

The horizontal projection was always abnormal. The first part of the loop showed a smooth curve (up to $0.02-0.03 \mathrm{sec}$.) but this section was mostly rotated forwards in an anti-clockwise direction (Fig. 2H). This first part of the loop is shown in Fig. 4 for each case in the series. It will be

* This work was completed when one of us (J.P.S.) was in receipt of a Medical Research Council grant. 

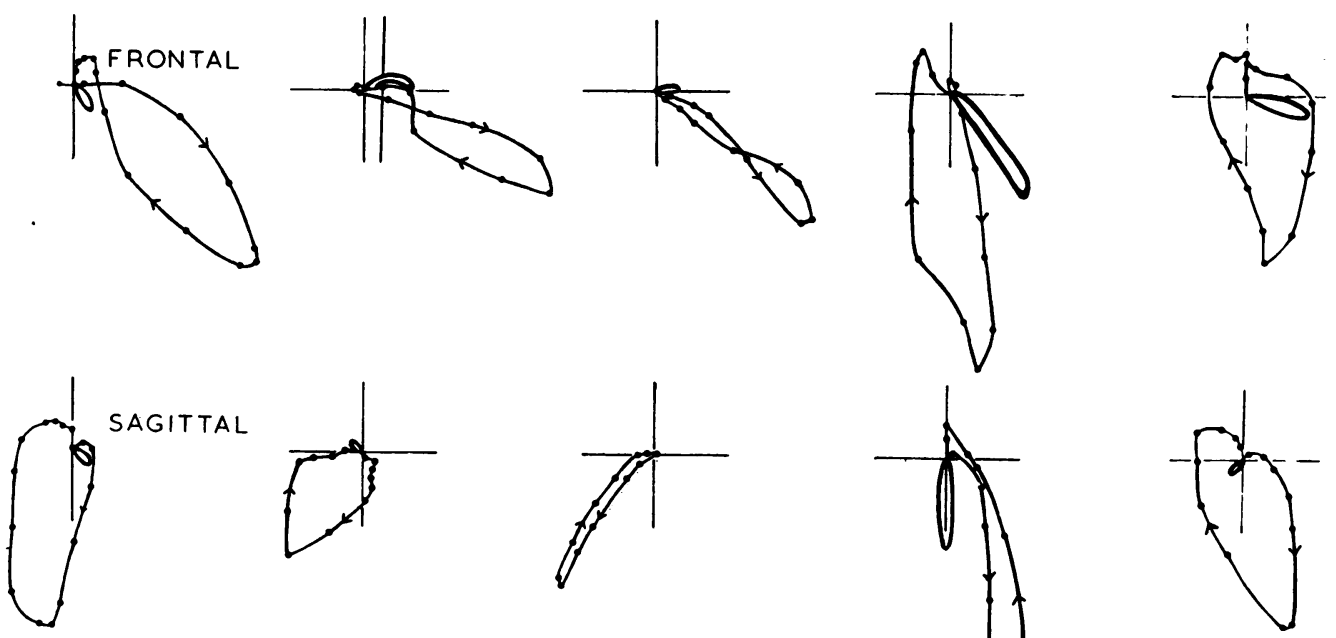

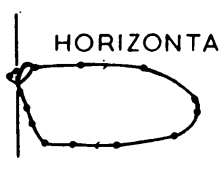

NORMAL

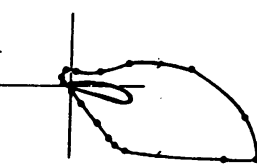

A

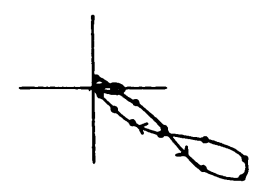

B
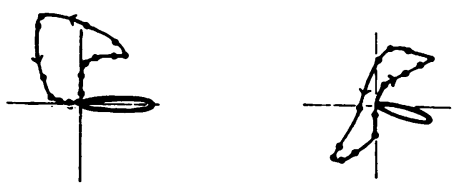

C

D

Fig. 1. - The frontal, sagittal, and horizontal projections of the spatial vectorcardiogram in the normal subject and in four cases of mitral stenosis. (A) Case without pulmonary hypertension or cardiac failure. (B) Case with congestive cardiac failure and much cardiac enlargement. (C) and (D) Cases with severe pulmonary hypertension and slight to moderate cardiac enlargement.

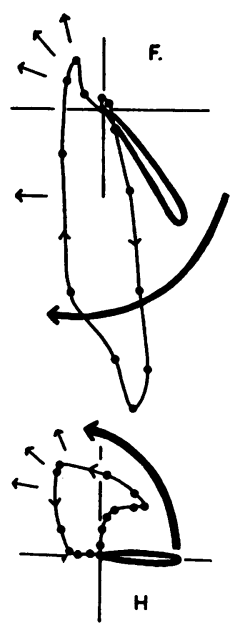

Fig. 2.-The frontal (F), sagittal (S), and horizontal (H), QRS and T loops in a case of mitral stenosis with pulmonary hypertension, showing direction of rotation (thick arrows) and deviation of the loop (thin arrows).

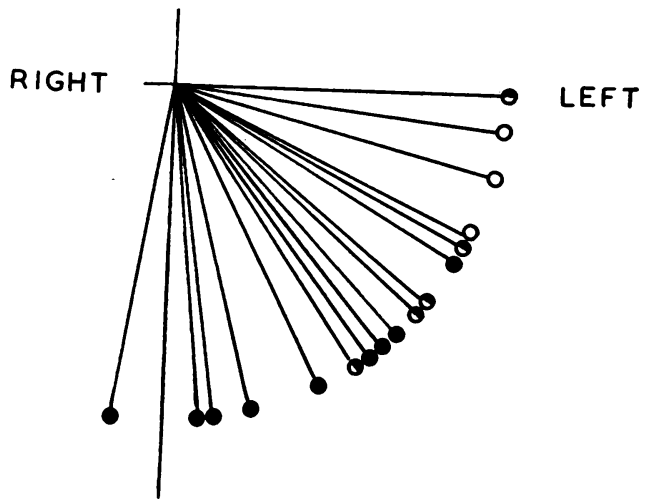

Fig. 3.-Diagram to illustrate the axis deviation as shown in the frontal loop in 17 patients with mitral stenosis.

With pulmonary hypertension.

- With congestive cardiac failure.

With almost normal hæmodynamics. 
seen that the anti-clockwise rotation particularly affects the pulmonary hypertensive group. As in the frontal loop there is a variable pathological deviation to the right of the later part of the loop, in addition to the rotation. The smooth curve of the first part of the loop was often abruptly changed between $0.02-0.03 \mathrm{sec}$. by this deviation to the right and, in a few cases, the greater part of the loop was found to lie to the right of the anteroposterior iso-electric line.

These abnormalities affected all the cases with pulmonary hypertension in varying degrees; no attempt was made to relate the degree of abnormality with the severity of the clinical lesion within this group.

The duration of the ventricular loop, excluding the $T$ component, was measured in all cases. No case showed an abnormal prolongation of the total time taken for inscription of the loop (and this included cases that showed RsR complexes in rightsided chest leads), and the average total time for the whole group was rather less than normal. A sharp deviation of the loop in the horizontal plane was frequently seen following the short initial curve; the duration of these early vectors from the

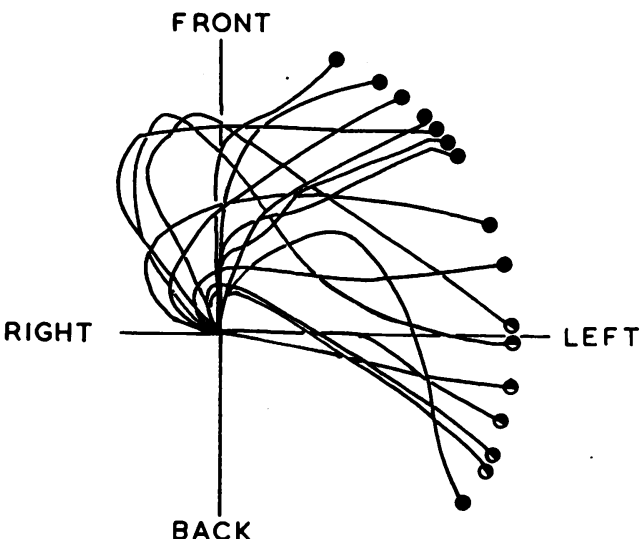

FIG. 4.-The septal part of the horizontal loops in 16 patients with mitral stenosis. Anti-clockwise rotation is present in most of the cases that showed pulmonary hypertension.

- Pulmonary hypertension.

Almost normal hæmodynamics. iso-electric point to the point of this deviation was measured and varied between 0.015 and $0.03 \mathrm{sec}$. This phase corresponds with the early electrical activity recorded on the electrocardiogram as a

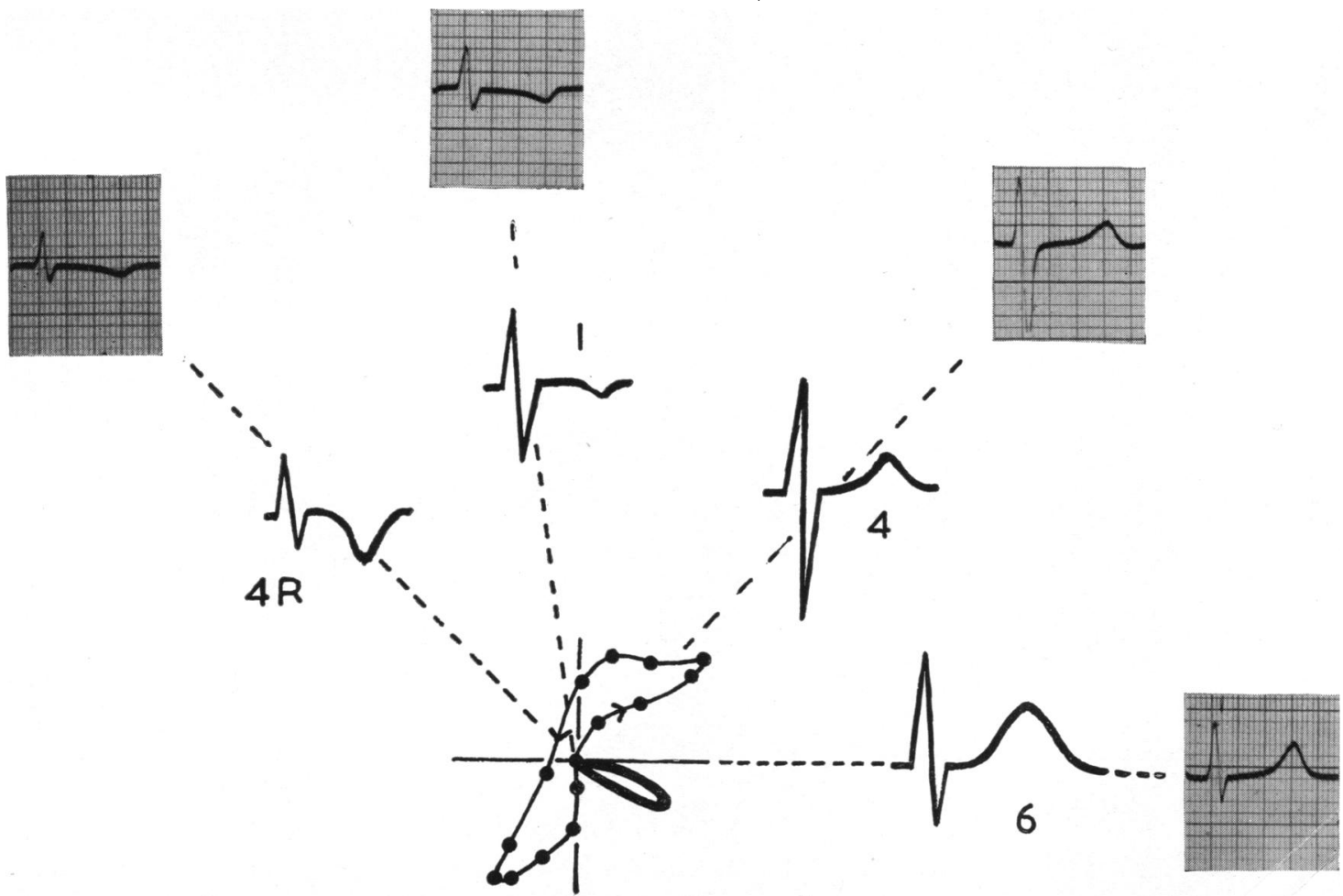

Fig. 5.-The horizontal loop from a case of mitral stenosis with pulmonary hypertension together with reconstructed chest leads and with $V$ chest leads. 
small $\mathrm{Q}$ wave in left chest leads or a small $\mathrm{R}$ in right-sided chest leads generally considered to be the result of septal excitation. The time taken from the iso-electric point to the point of maximal deviation to the right was measured in those cases showing such deviation. This time interval was relatively constant, being between 0.05 and $0.055 \mathrm{sec}$., and was unrelated to any variation in the total QRS loop time, which ranged from 0.06 to $0.075 \mathrm{sec}$.

\section{The RELATIONShiP Between VectorCardiograms and Electrocardiograms}

There was a close relationship between QRS-T complexes reconstructed from the loops and the actual complexes recorded on standard bipolar leads and unipolar limb and chest leads. The qualitative form was very similar in all cases and in all lead positions. When due allowance is made for the effects of the inverse square law, the chest leads closely resembled their reconstructed counterparts (Fig. 5).

All the vectorcardiograms that showed a frontal loop with its largest vector directed more or less vertically (i.e. showing a degree of rotation to the right in this plane) also showed related degrees

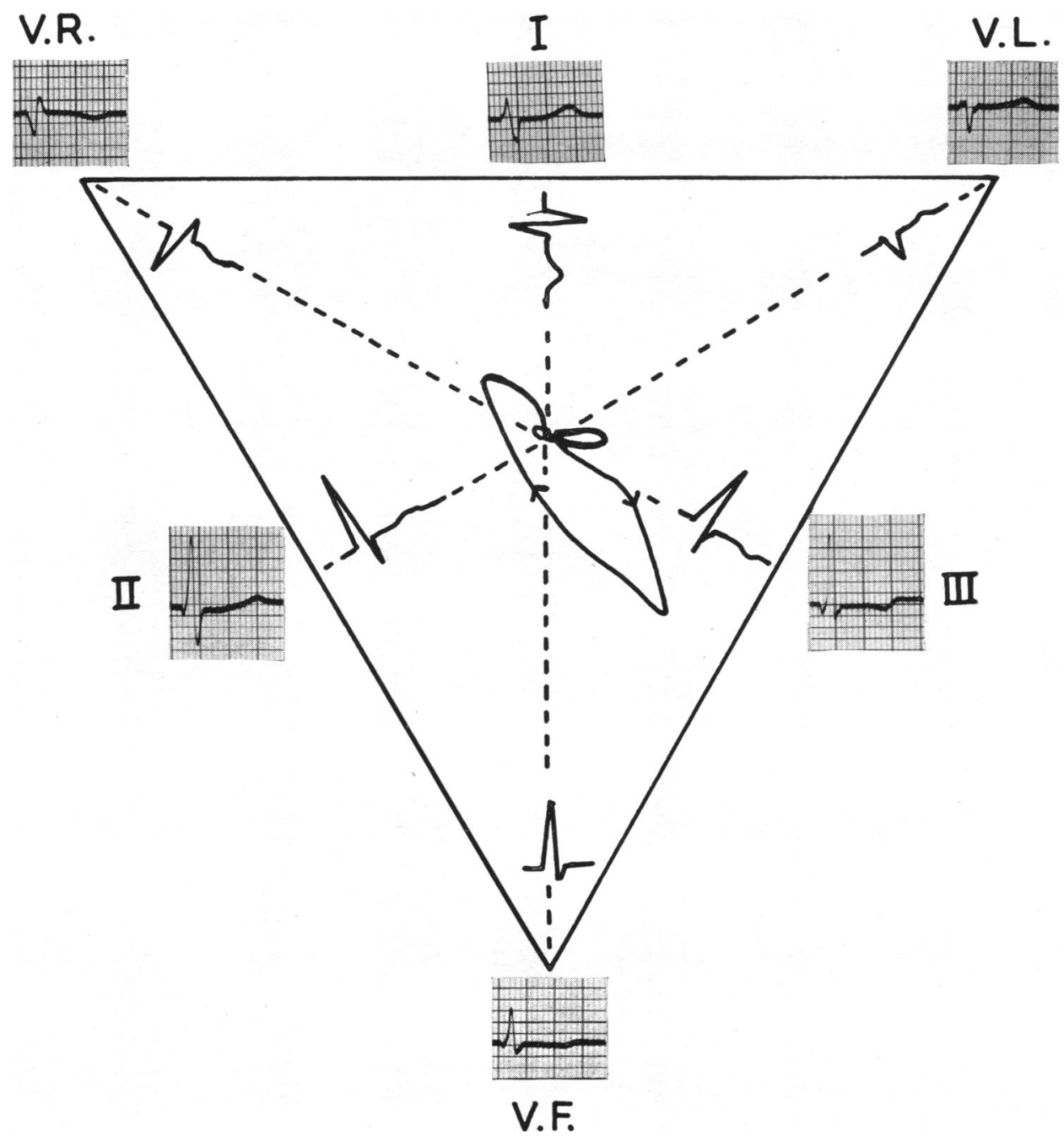

FIG. 6.-The frontal loop from a patient with mitral stenosis and pulmonary hypertension with parts of reconstructed and recorded bipolar and unipolar limb leads. 
of right axis deviation in the standard limb leads. Frontal vectorcardiograms having some deformity of the returning limb of the loop into the right lower and particularly right upper quadrants also showed a tall secondary $R$ wave in lead VR (Fig. 6). These changes of axis deviation and of lead VR were most apparent in the patients with pulmonary hypertension and were considered to be an expression of right ventricular hypertrophy.

The chest lead electrocardiograms in these cases tend to show RS complexes well over to the left side and this is frequently interpreted as clockwise rotation (when viewed from below). Indeed this is considered to be a characteristic of the electrocardiogram of mitral stenosis by some authors. However, the horizontal loops of the vectorcardiogram showed that the septal or early part of the loop is rotated if anything in an anti-clockwise direction (Fig. 4 and 5) and the rest of the loop is deviated in a pathological form to the right side: thus there is no evidence of clockwise rotation in the vectorcardiograms of these cases of mitral stenosis. In spite of this

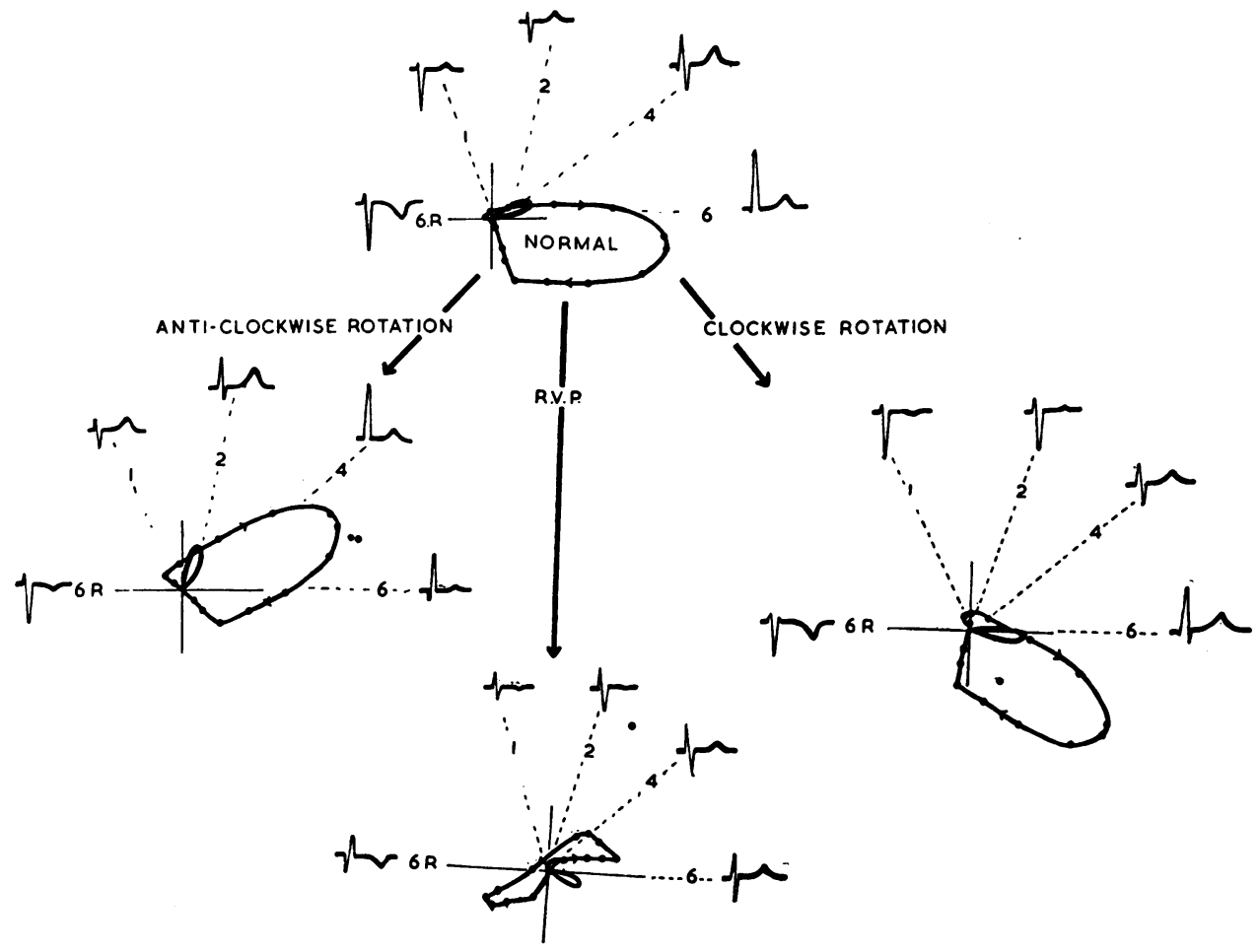

Fig. 7.-The horizontal projection of the vectorcardiogram and corresponding electrocardiograms in (1) anti-clockwise rotation, (2) clockwise rotation, (3) right ventricular preponderance, and their relationship to the normal.

apparent paradox, it was found that the correlation of unipolar leads across the chest from V6R to V6 with the lead patterns reconstructed from the horizontal loops was remarkably close. It seems, therefore, that the RS complexes appearing in the left chest leads and accompanied by a shift to the left of the maximum height of the $T$ wave, and interpreted by some as the effect of clockwise rotation, is the result of the pathological deviation of vectors to the right side and is quite unrelated to any effect of rotation. Reference to Fig. 7 shows how the same RS pattern can be either the result of right ventricular preponderance or clockwise rotation and in this series all the patients showing so-called clockwise rotation electrocardiograms were, in fact, those showing right ventricular preponderance on the loop.

These abnormal horizontal loops were redrawn on semi-transparent paper and the point of zero C 


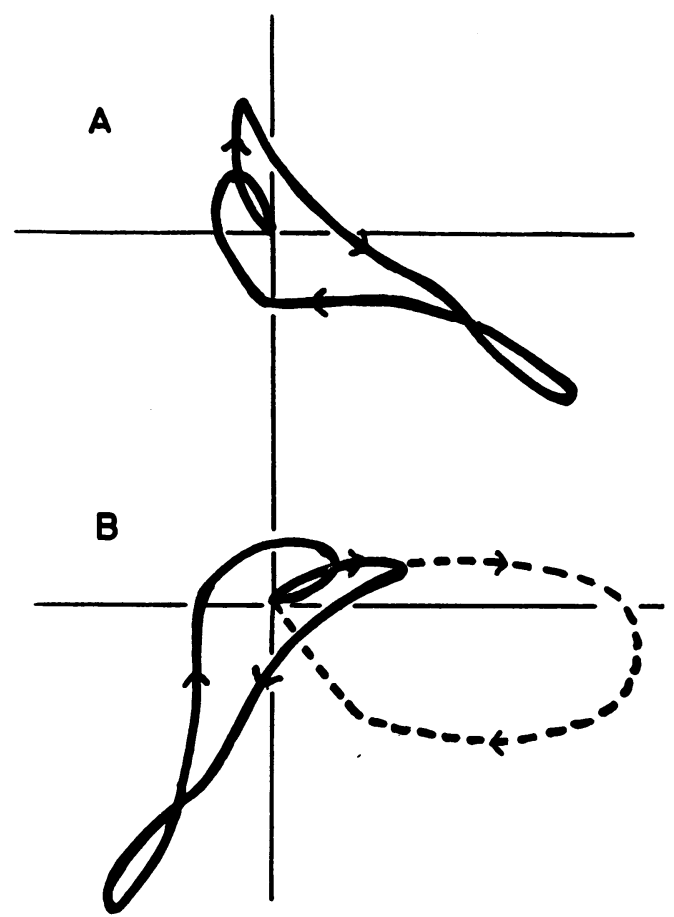

Fig. 8.-(A) Horizontal loop from a patient with mitral stenosis and severe pulmonary hypertension. (B) Loop rotated until septal vectors lie in the position of a normal loop-the greatest vectors are now directed to the right side. Dotted loop shows the normal pattern. monary hypertension. There was no relation between the abnormalities of the ventricular vectorcardiogram and the presence or absence of auricular flbrillation. The determining factor in changing
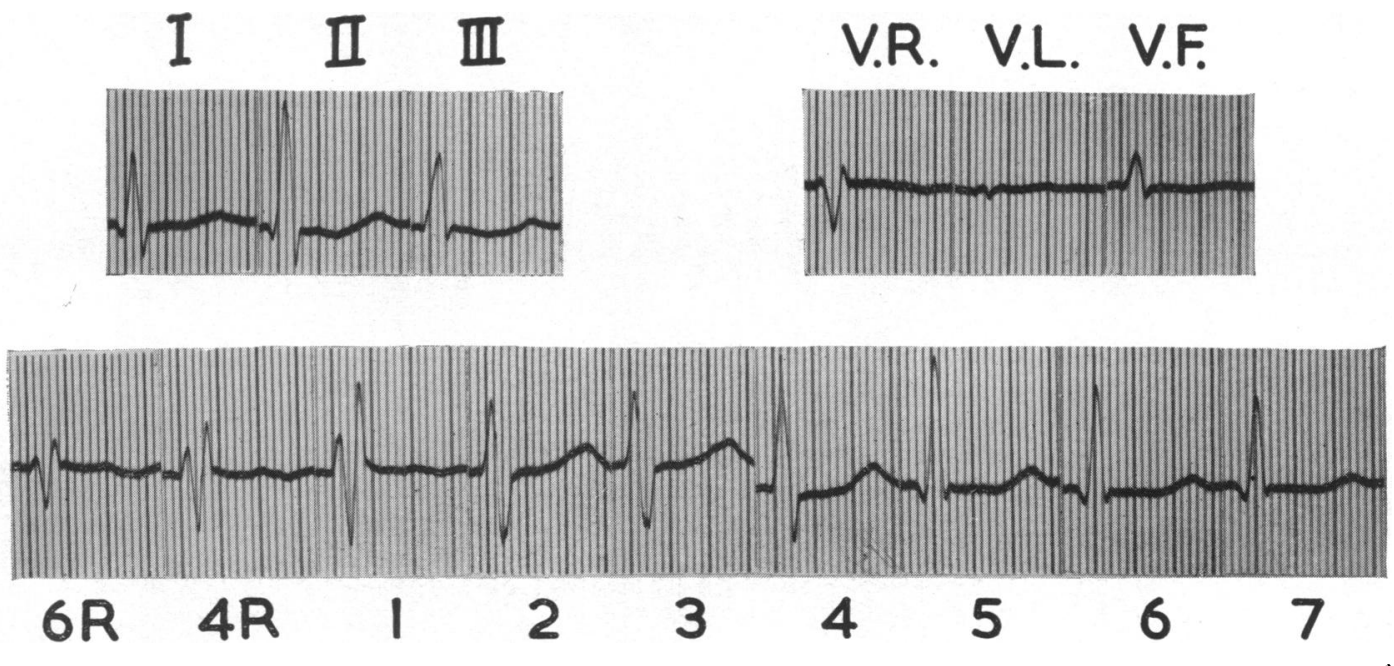

FIG. 9.-Electrocardiogram of the patient whose horizontal vectorcardiogram is shown in Fig. 8.

potential applied to the zero of a normal loop by rotating the abnormal loop in a clockwise direction until the septal parts were roughly superimposed and the rotation effect was "corrected." When this was done the pattern of right ventricular preponderance became more apparent (Fig. 8).

All of the patients with pulmonary hypertension showing pathological right-side deviation of the horizontal loop in some degree also showed an increase in their positive deflection in right chest leads V4R and V6R. The horizontal loops from patients having an rSR complex in V1 showed the initial vectors directed towards this position and later vectors also directed to this position (Fig. 8A and 9). In these cases there was also close agreement between reconstructed leads and $\mathrm{V}$ chest leads.

The $\mathrm{T}$ loop showed rotation in a clockwise direction associated with the development of right ventricular hypertrophy, thus producing a shift in the increase of height of the $T$ waves to the left in conventional leads. This tendency was counteracted in many cases by the anti-clockwise rotation of the vectorcardiogram as a whole.

\section{Discussion AND CONCLUSIONS}

The greatest changes in the vectorcardiogram in mitral stenosis were found in cases with pul- 
the shape of the vectorcardiogram, therefore, seemed to be the development of pulmonary hypertension and consequent hypertrophy of the right ventricle.

In all views of the loop the pathological changes showed as a shift or rotation of the whole loop and as local deviation of the loop due to preponderance of different parts of the heart at particular phases in the cardiac cycle. In the frontal projection the appearance of the loop was similar to that produced by pulmonary hypertension from other causes. In this projection the loop tends to rotate to a more vertical position, the counterpart of right-axis deviation. In each case, the most abnormal vectorcardiograms from those with pulmonary hypertension were also associated with electrocardiograms showing the well-known pattern of an S in lead I and an $\mathrm{R}$ in lead III. The local deviation of the frontal loop into the right lower and upper quadrants was found in the most abnormal loops and was invariably associated with a tall late $R$ wave in lead VR emphasizing the importance of this lead in the diagnosis of right ventricular hypertrophy.

The abnormalities of the horizontal loop also appear to be due to right ventricular hypertrophy and rotation. It is generally accepted that the first $0.02 \mathrm{sec}$. of the QRS represents excitation of the ventricular septum. It is thus probable that the early part of the loop $(0.02 \mathrm{sec}$.) is due to the same cause, and deviation of this first part of the loop in the horizontal plane may be interpreted as a rotation of the plane of the septum and of the electrical field as a whole. A gross deviation of the loop from its normal smooth contour after this initial phase may be due to pathological preponderance of either right or left ventricle. In this series, where pulmonary hypertension was present, the rotation of the first segment of the loop was always in an anti-clockwise direction while the alterations beyond this point showed a deviation of the vector to the right side. It is usually stated from an examination of the conventional electrocardiographic leads that clockwise rotation is present in mitral stenosis but this impression seems to be incorrect and it is produced by right ventricular preponderance combined with the less important effects of anti-clockwise rotation. The cause of this rotation is not known but it may be related to the anatomical position of the tense left atruim; it is unlikely to be due to a high pressure in the pulmonary artery with consequent unfolding of this vessel for in other forms of pulmonary hypertension we have not observed anti-clockwise rotation. In some cases the anti-clockwise rotation was so great as to minimize the appearance of right ventricular preponderance on the conventional electrocardiogram. This effect is shown in Fig. 8A and 9 where the standard electrocardiogram appeared to show a slight degree of right ventricular hypertrophy without S waves in V7. When the horizontal loop of the vectorcardiogram was studied, however, it was seen to show great anti-clockwise rotation which, when " corrected," placed the bulk of the vectorial forces to the right and reconstructions from this loop show tall $R$ waves in right chest leads and $S$ waves in left chest leads. It is well known that the electrocardiographic features of right ventricular preponderance are often unimpressive in the routine leads in cases of mitral stenosis even when some degree of pulmonary hypertension is known to be present; it seems possible that anti-clockwise rotation is responsible for this.

Unipolar electrocardiograms from the right side of the chest showed a pathological increase in $\mathrm{R}$ waves in all cases where the horizontal loop was pathological and we consider that V4R or V6R should be recorded in cases of suspected right ventricular hypertrophy. CRR leads should not be used in these cases since it was found that as the exploring electrode approached the right side (and therefore the right arm) there was increasing discrepancy between these patterns and those obtained from recorded unipolar leads or those reconstructed from the horizontal loop. In some cases showing great anti-clockwise rotation where the first part of the horizontal loop was directed towards the V1 position and at the same time the main part of the loop was deviated to the right so that a second set of vectors were directed towards the V1 position, an rSR pattern was produced. This pattern repiesents right ventricular preponderance and differs from that of right bundle branch block of the Wilson type (Lasser et al., 1951) although the scalar electrocardiograms are similar.

The time of appearance of the maximum vectors to the right bore a remarkably constant time relation to the commencement of the loop in the presence of right-sided preponderance and occurred 
at a time of $0.05-0.55 \mathrm{sec}$. from the iso-electric point. This time interval was unrelated to the total time of the ventricular loop and its significance is not clear.

The vectorcardiogram appeared to give a better overall picture of right ventricular hypertrophy in mitral stenosis than the conventional electrocardiogram, although nearly as much information could be gathered from a study of the standard limb leads and chest leads when these included VR and V4R. The advantage contributed by the vectorcardiogram was the clear distinction between hypertrophy, rotation, and the different rSR patterns.

\section{SUMMARY}

Three plane projections of the vectorcardiogram have been drawn in 20 cases of mitral stenosis. Those patients with severe pulmonary hypertension and slight to moderate cardiac enlargement showed gross abnormalities of the vectorcardiogram due to right heart preponderance. There was no evidence of clockwise rotation and the persistent RS pattern found in left chest leads in mitral stenosis was due to right heart preponderance. An rSR pattern in right chest leads was also associated with the vectorcardiogram of right heart preponderance.

The relation between the vectorcardiogram and the electrocardiogram in mitral stenosis has been discussed. The vectorcardiogram gives a clearer distinction between the factors of hypertrophy and rotation and the different rSR patterns.

\section{REFERENCES}

Brigden, W., and Shillingford, J. (1952). Brit. Heart J., 14, 339.

Lasser, R. P., Bonur, E. R., and Grishman, A. G. (1951). Amer. Heart J., 41, 667. 